\title{
A non-randomised, single-centre comparison of induction chemotherapy followed by radiochemotherapy versus concomitant chemotherapy with hyperfractionated radiotherapy in inoperable head and neck carcinomas \\ Reinhold Graf1 ${ }^{1}$, Bert Hildebrandt ${ }^{2}$, Wolfgang Tilly¹, Hanno Riess ${ }^{2}$, Roland Felix ${ }^{1}$, Volker Budach ${ }^{3}$ and Peter Wust*1
}

\begin{abstract}
Address: ${ }^{1}$ Clinic for Radiation Medicine, Charité Medical School, Campus Virchow-Klinikum, Augustenburger Platz,113353 Berlin, Germany, ${ }^{2}$ Medical Clinic for Hematology and Oncology, Charité Medical School, Campus Virchow-Klinikum, Augustenburger Platz 1, 13353 Berlin, Germany and ${ }^{3}$ Clinic for Radiation Oncology, Charité Medical School, Campus Charité Mitte, Schumannstr. 21, 10117 Berlin, Germany

Email: Reinhold Graf - reinhold.graf@charite.de; Bert Hildebrandt - bert.hildebrandt@charite.de; Wolfgang Tilly - wolfgang.tilly@charite.de; Hanno Riess - hanno.riess@charite.de; Roland Felix - roland.felix@charite.de; Volker Budach - volker.budach@charite.de;

Peter Wust* - peter.wust@charite.de

* Corresponding author
\end{abstract}

Published: 0 I February 2006

BMC Cancer 2006, 6:30 doi:10.1186/147|-2407-6-30

This article is available from: http://www.biomedcentral.com/I47/-2407/6/30

(c) 2006 Graf et al; licensee BioMed Central Ltd.

This is an Open Access article distributed under the terms of the Creative Commons Attribution License (http://creativecommons.org/licenses/by/2.0), which permits unrestricted use, distribution, and reproduction in any medium, provided the original work is properly cited.
Received: 02 August 2005

Accepted: 01 February 2006

\begin{abstract}
Background: The application of induction chemotherapy failed to provide a consistent benefit for local control in primary treatment of advanced head and neck $(\mathrm{H} \& \mathrm{~N})$ cancers. The aim of this study was to compare the results of concomitant application of radiochemotherapy for treating locally advanced head-and-neck carcinoma in comparison with the former standard of sequential radiochemotherapy.
\end{abstract}

Methods: Between 1987 and 1995 we treated 122 patients with unresectable (stage IV head and neck) cancer by two different protocols. The sequential protocol (SEQ; 1987-1992) started with two courses of neoadjuvant chemotherapy (cisplatin [CDDP] + 120-h continuous infusions (c.i.) of folinic acid [FA] and 5-fluorouracil [5-FU]), followed by a course of radiochemotherapy using conventional fractionation up to $70 \mathrm{~Gy}$. The concomitant protocol (CON; since 1993) combined two courses of FA/5-FU c.i. plus mitomycin (MMC) concomitantly with a course of radiotherapy up to $30 \mathrm{~Gy}$ in conventional fractionation, followed by a hyperfractionated course up to $72 \mathrm{~Gy}$. Results from the two groups were compared.

Results: Patient and tumor characteristics were balanced (SEQ $=70, \mathrm{CON}=52$ pts.). Mean radiation dose achieved $(65.3 \mathrm{~Gy}$ vs. $71.6 \mathrm{~Gy}, \mathrm{P}=0.00)$, response rates (67 vs. $90 \%$ for primary, $\mathrm{P}$ $=0.02$ ), and local control (LC; $17.6 \%$ vs. $41 \%, p=0.03$ ), were significantly lower in the SEQ group, revealing a trend towards lower disease-specific (DSS; 19.8\% vs. $31.4 \%, p=0.08)$ and overall $(I 4.7 \%$ vs. $23.7 \%, p=0.1 \mathrm{I})$ survival rates after 5 years. Mucositis grades III and IV prevailed in the CON group (54\% versus $44 \%$ ). Late toxicity was similar in both groups.

Conclusion: Concurrent chemotherapy seemed more effective in treating head and neck tumors than induction chemotherapy followed by chemoradiation, resulting in better local control and a trend towards improved survival. 


\section{Background}

Various approaches have been developed to intensify standard radiotherapy in locally advanced head and neck carcinomas, such as altered fractionation schemes (hyperfractionation, acceleration) [1] or combination with chemotherapy [2]. Improved local control and survival have been confirmed in recent trials of concomitant radiochemotherapy [2-4]. However, three meta-analyses of radiochemotherapy in advanced head and neck cancer [5] showed that the impact of chemotherapy on overall survival is rather small (improving e.g. 5-year overall survival from $30 \%$ to $35 \%$ ). Compared to radiotherapy alone, concomitant radiochemotherapy shows the biggest improvement with respect to local control and diseasespecific survival. As a result, concomitant radiochemotherapy with cisplatin and 5-FU is increasingly accepted as standard treatment for patients with unresectable head and neck carcinoma and adequate performance status.

To date, no randomised study has compared induction chemotherapy followed by concurrent radiotherapy against chemotherapy with a concomitant radiochemotherapy schedule. We present such a non-randomised comparison with respect to late toxicity, local control and survival in patients with stage IV unresectable head and neck cancers, who were treated according to two different schedules at our institution between 1987 and 1995.

\section{Methods}

Between March 1987 and July 1995, 122 patients were treated with two consecutive phase-II protocols in the departments of radiation oncology and hematology/
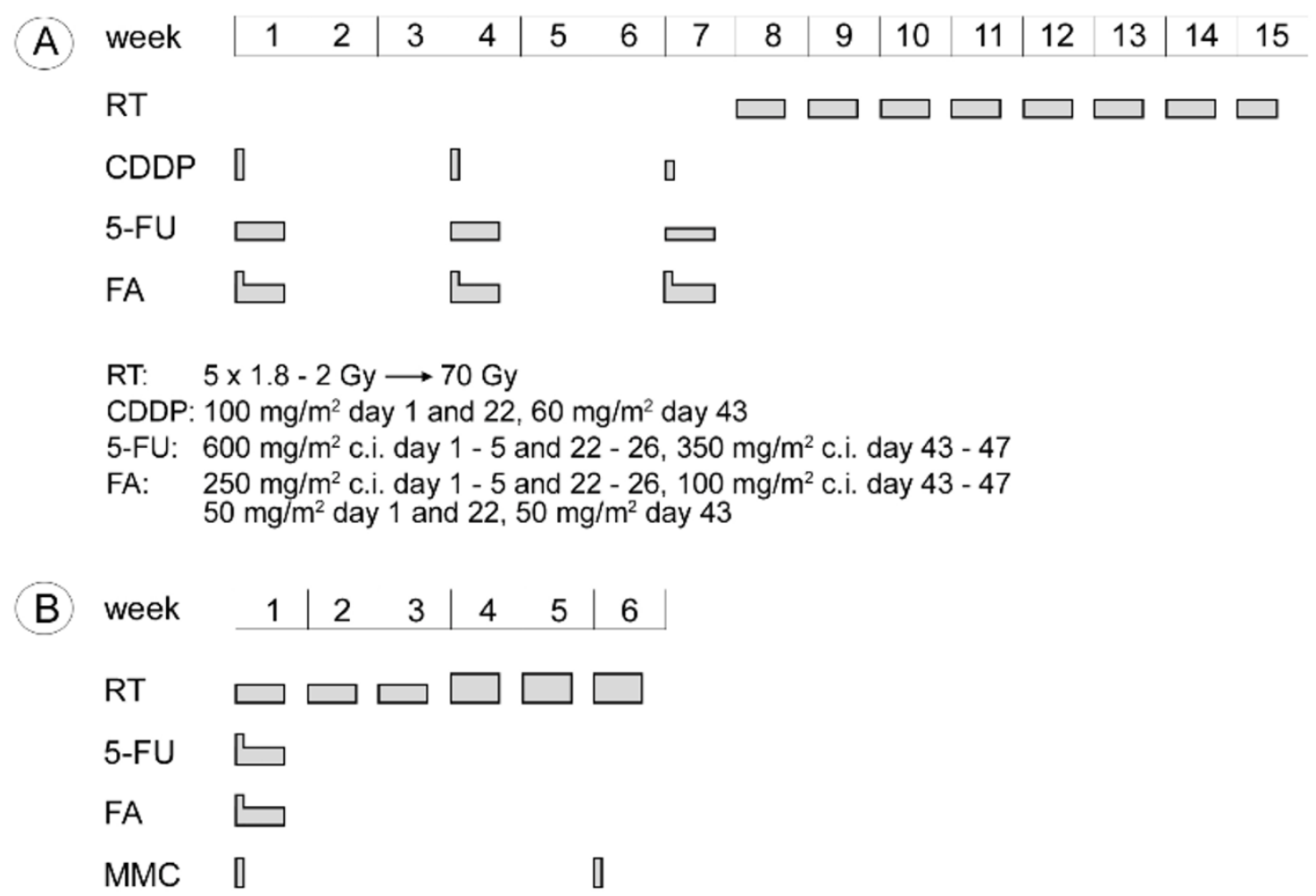

RT: $\quad 5 \times 2$ Gy week $1-3,5 \times 2 \times 1.4$ Gy week 4 - 6

5-FU: $350 \mathrm{mg} / \mathrm{m}^{2}$ c.i. day $1-5$,

$$
50 \mathrm{mg} / \mathrm{m}^{2} \text { day } 1
$$

FA: $\quad 100 \mathrm{mg} / \mathrm{m}^{2}$ c.i. day $1-5$, $50 \mathrm{mg} / \mathrm{m}^{2}$ day 1

MMC: $10 \mathrm{mg} / \mathrm{m}^{2}$ day 1 and 36

\section{Figure I}

Flow sheet of the two treatment regimes. A (top): treatment schema, sequential protocol (SEQ.). B (bottom): treatment schema, concomitant protocol (CON). 
oncology at the Charité. The first, sequential (SEQ) protocol was performed between 1987 and 1992 and included neoadjuvant chemotherapy with a subsequent radiotherapy course. The second protocol was performed after 1992 and consisted of concomitant radiochemotherapy with subsequent hyperfractionation.

The first protocol was approved by the ethical committee at the Virchow-Klinikum and the second by the ethical committee of the Campus Charité-Mitte of the Charité.

\section{Eligibility criteria}

Patients were eligible if they had primary, histologically proven, non-metastatic squamous cell carcinoma of the oral cavity, oropharynx, hypopharynx or larynx, assessed as unresectable in the department of head-and-neck surgery because of size, extension or location. Patients who had previously undergone cytostatic therapy or radiotherapy were excluded, along with those with contraindications against radiotherapy or the cytostatic agents used (5FU, FA, CDDP, MMC). Written informed consent was obtained from all patients before therapy.

\section{Evaluation of patients}

For every patient, clinical, laboratory and imaging examinations were performed to achieve an accurate TNM stage (UICC, $5^{\text {th }}$ ed.) [6] and to allow follow-up.

During therapy, the patients were examined for acute toxicity every week. Acute side effects were indexed according to EORTC criteria [7]; late side effects were classified according to the LENT tables [8]. The response (primary as well as lymph nodes) was assessed by clinical examination and CT according to WHO criteria [9].

After completion of therapy, each patient was followed up within $6-8$ weeks by an interdisciplinary consultation involving clinical evaluation (head and neck status) complemented by CT. After specific symptoms or suspicious findings, diagnostic procedures were extended. Further follow-up included examinations at intervals extending from 6 weeks initially to $6-12$ months after the third year.

In cases of histologically proven local recurrence, all available salvage treatment options were offered (brachytherapy, hyperthermia, external re-irradiation, chemotherapy); most often palliative chemotherapy with MTX (methotrexate) was performed.

\section{Treatment records (fig. $I A+B$ )}

In the first sequential (SEQ) protocol, 2 three-weekly courses of neoadjuvant chemotherapy were applied, consisting of cisplatin (CDDP; $100 \mathrm{mg} / \mathrm{m}^{2}$ i.v., on day 1) followed by $50 \mathrm{mg} / \mathrm{m}^{2}$ folinic acid (day 1 ) and parallel 120 h continuous infusions of folinic acid $\left(250 \mathrm{mg} / \mathrm{m}^{2}\right.$ per day, days 1-5) and 5-fluorouracil (600 mg/m², days 1-5). Six weeks later (day 43), the third chemotherapy course was started at a $60 \%$ reduced dose concomitantly with the definitive radiotherapy. External beam radiotherapy was applied without preference to both groups, with photon energies of $1.2 \mathrm{MeV}$ (telecobalt unit) or 4-8 MV (linear accelerator). The primary tumor and regional lymph drainage areas received $60 \mathrm{~Gy}$ in single fractions of 1.8-2 Gy. To shield the spinal cord after achieving a dose of 40 $\mathrm{Gy}$, the dorsal parts of the target volume were treated with fast electrons of 9-12MeV. Primary tumor and lymph node areas that were macroscopically involved were boosted to a total dose of $70 \mathrm{~Gy}$.

In the second, concomitant (CON) schedule, an RCT protocol was conducted concomitantly with chemotherapy as in the study of Budach $[4,10]$. Here, chemotherapy was initiated with intravenous short infusions of folinic acid $\left(50 \mathrm{mg} / \mathrm{m}^{2}\right.$, day 1$)$ and 5 -FU $\left(350 \mathrm{mg} / \mathrm{m}^{2}\right.$, day 1$)$, followed by parallel $120 \mathrm{~h}$ continuous infusions with both drugs (folinic acid, $100 \mathrm{mg} / \mathrm{m}^{2}$ daily; 5 -FU, $350 \mathrm{mg} / \mathrm{m}^{2}$ daily). In addition, short infusions of mitomycin C (10 $\mathrm{mg} / \mathrm{m}^{2}$ ) were administered on days 1 and 36. Radiotherapy began synchronously with chemotherapy, starting with single doses of 2 Gy up to a total dose of 30 Gy over the entire affected volume. Gross areas were then boosted using hyperfractionation, with two daily 1.4 Gy fractions separated by an interval of at least 6 hours, to a total dose of 70.6 Gy (10 patients) or 72 Gy (42 patients). Lymph node regions at low or intermediate risk of subclinical infiltration were irradiated to total doses of $50 \mathrm{~Gy}$ (normofractionated) or 60 Gy (hyperfractionated after $30 \mathrm{~Gy}$ ), respectively. The dorsal paravertebral parts of the target volume were irradiated with lateral 6-9 MeV electron fields after 36-40 Gy at the myelon, conventionally fractionated if not clinically involved, hyperfractionated otherwise.

\section{Statistical evaluation}

We used the distribution-free Mann-Whitney test to compare the two patient groups (SEQ/CON). A univariate analysis of prognostic factors was carried out through a comparison of survival times and local control rates using the log-rank test. A multivariate analysis was carried out for all parameters with significant correlations in the univariate analysis according to the proportional-hazard model. Time-to-progression (TTP), tumour specific and overall survival were identified from the first day of treatment up to the day of death or last patient contact following the Kaplan-Meier method [11]. In particular, TTP was defined as the time from day 1 of treatment until clinical progression, i.e. the timing of a more than $25 \%$ increase in post-therapeutic volume (determined 6-8 weeks after 
Table I: Comparison of patients' characteristics differentiated to the type of schedule

\begin{tabular}{|c|c|c|c|c|c|}
\hline \multirow[b]{2}{*}{ Parameter } & \multicolumn{2}{|c|}{ SEQ (sequential, $n=70$ ) } & \multicolumn{2}{|c|}{ CON (concomitant, $n=52)$} & \multirow{2}{*}{$\begin{array}{l}\mathbf{P} \\
0.80\end{array}$} \\
\hline & number & (\%) & number & $(\%)$ & \\
\hline \multicolumn{6}{|l|}{ Age, years } \\
\hline Mean (Range) & $53(37-77)$ & & $52.8(39-66)$ & & 0.79 \\
\hline Performance status (WHO) & & & & & 0.67 \\
\hline 0,1 & 35 & $(50)$ & 30 & $(58)$ & \\
\hline 2,3 & 27 & (39) & 19 & (37) & \\
\hline Unknown & 8 & (II) & 2 & (4) & \\
\hline T-stage (UICC) & & & & & 0.54 \\
\hline T2 & 4 & (6) & 3 & (6) & \\
\hline T3 & 18 & $(26)$ & 9 & $(17)$ & \\
\hline T4 & 48 & $(68)$ & 40 & (77) & \\
\hline N-stage & & & & & 0.13 \\
\hline $\mathrm{N} 2 \mathrm{c}$ & 23 & $(33)$ & 15 & (29) & \\
\hline N3 & 8 & (II) & 7 & (14) & \\
\hline RTOG stage groupings & & & & & 0.58 \\
\hline III (T3-4 N0, TI-2 N2) & 5 & (7) & I & $(2)$ & \\
\hline IV (TI-2 N3, T3 NI-2, T4 NI) & 23 & $(33)$ & 16 & (3I) & \\
\hline $\mathrm{V}(\mathrm{T} 4 \mathrm{~N} 2-3)$ & 41 & (59) & 35 & $(67)$ & \\
\hline \multicolumn{6}{|l|}{ UICC stage groupings } \\
\hline $\mathrm{IV}(\mathrm{a}+\mathrm{b})$ & 69 & (99) & 52 & $(100)$ & \\
\hline Tumor location & & & & & 0.19 \\
\hline Oral cavity & 7 & $(10)$ & 10 & $(19)$ & \\
\hline Oropharynx & 38 & $(54)$ & 30 & (58) & \\
\hline Hypopharynx, Larynx & 25 & $(36)$ & 12 & (23) & \\
\hline Mean tumor size $(\mathrm{cm})$ & 4.8 & & 4.6 & & 0.46 \\
\hline
\end{tabular}

end of treatment) in any tumor lesion, or histopathological validation of recurrent disease after a CR.

\section{Results}

Seventy patients in the SEQ group and 52 patients in the CON group were included in an intent-to-treat analysis. The median observation times for the surviving patients were 53 months for the SEQ group and 41 for the CON group, respectively (for all patients, 19.8 and 14.7 months in the two groups).

\section{Patients' characteristics}

We found a comparable distribution of prognostic factors in both groups with regard to age, gender and performance status (WHO). Tumors of the oropharynx predominated, with frequencies of $54 \%$ and $58 \%$ in the SEQ and CON group, respectively $(\mathrm{p}=0.66)$. The percentage of tumors located in the hypopharynx was found to be slightly higher in the SEQ group (29\% vs. $17 \%$ of patients, $\mathrm{p}=0.13$ ). All other tumor characteristics were statistically equally distributed according to table 1 . Most patients had advanced stages, with T4-categories in $68 \%$ vs. $77 \%(\mathrm{p}=$ 0.28 ), and N2c-N3 in $44 \%$ vs. $43 \%$, of patients in the SEQ vs. CON groups, respectively. The resulting clinical stages were as high as IV and V (RTOG) for the majority of the patients, i.e. $33 \%$ and $59 \%$ (together $92 \%$ ) for the SEQ group and $31 \%$ and $67 \%$ (together $98 \%, p=0.37$ ) for the CON group.

\section{Treatment parameters}

In the SEQ group, $5 / 70$ patients $(7 \%)$ received only one chemotherapy course owing to rapid tumor progression and/or poor performance status. Two courses were applied to $22 / 70$ patients (31\%), and three cycles were given to only $43 / 70(62 \%)$. Of these, $65 / 70(95 \%)$ could be evaluated for response after induction chemotherapy, and $62 / 70(89 \%)$ after the whole treatment course. Patients who could not be evaluated for response included three treatment-related deaths during the first two cycles. At the second checkpoint (after radiotherapy), 4/70 patients were non-evaluable and received no radiotherapy.

In the CON group, the first course of chemotherapy was given to all patients with at least $80 \%$ of the prescribed 5FU dose level. Two of the patients received only one application of mitomycin $\mathrm{C}$ in week 1 because the whole treatment had resulted in considerable toxicity by week 6 . 
Table 2: Acute and late toxicity after radiotherapy and chemotherapy according to RTOG/ECOG and LENT scores (highest level given)

\begin{tabular}{|c|c|c|c|c|c|c|c|c|c|}
\hline & \multicolumn{4}{|c|}{ SEQ } & \multicolumn{5}{|c|}{ CON } \\
\hline & \multicolumn{2}{|c|}{ Grade III } & \multicolumn{2}{|c|}{ Grade IV } & \multicolumn{2}{|c|}{ Grade III } & \multicolumn{2}{|c|}{ Grade IV } & \multirow[t]{2}{*}{$\mathbf{p}$} \\
\hline & $\mathbf{n}$ & (\%) & $\mathbf{n}$ & (\%) & $\mathbf{n}$ & (\%) & $\mathbf{N}$ & (\%) & \\
\hline \multicolumn{10}{|c|}{ Acute toxicity (RTOG) } \\
\hline Skin & II & $(16)$ & 0 & & 5 & $(10)$ & 0 & & n.s. \\
\hline Mucositis & 31 & (44) & 1 & $(\mathrm{I})$ & 28 & $(54)$ & 2 & (4) & n.s. \\
\hline Pharynx & 9 & (13) & 1 & (I) & 9 & $(17)$ & I & (2) & n.s. \\
\hline Salivary glands & 10 & (14) & 0 & & 4 & (8) & 0 & & n.s. \\
\hline \multicolumn{10}{|c|}{ Late toxicity (LENT scores) } \\
\hline Skin & 2 & (3) & 0 & & 2 & $(4)$ & 0 & & n.s. \\
\hline Subcutis & 5 & (7) & 0 & & 7 & (14) & 0 & & n.s. \\
\hline Mucosa & 4 & (6) & 0 & & 3 & $(6)^{\prime}$ & 0 & & n.s. \\
\hline Salivary glands & 7 & (10) & 0 & & 3 & (6) & 0 & & n.s. \\
\hline Pharynx & 5 & (7) & 0 & & 4 & (8) & 1 & (2) & n.s. \\
\hline Larynx & 3 & (4) & 0 & & 2 & (4) & 0 & & n.s. \\
\hline Mandibula & 1 & (I) & 0 & & 0 & & 1 & (2) & n.s. \\
\hline
\end{tabular}

Only in $1 / 52$ of the patients $(2 \%)$ was radiochemotherapy abandoned, so $51 / 52$, i.e. $98 \%$, of the patients were further evaluable.

On average, we applied an average radiation dose of 65.3 Gy to the primary tumor in the SEQ group and 71.6 Gy in the CON group. The corresponding mean radiation doses at the involved lymph nodes were 58.1 Gy and 69.5 Gy, respectively. These doses were significantly different in the two groups $(\mathrm{p}=0.00)$.

In $23 / 61(36 \%)$ of the patients in the SEQ group, radiotherapy was interrupted for longer than 1 week, while 14/ $61(23 \%)$ had a break for longer than 3 weeks. In contrast, only $7 / 51(14 \%)$ of the patients in the CON group had a break of at least 1 week, and 3/51 (6\%) for more than 2 weeks $(p=0.00)$. In consequence, the average duration of radiotherapy (if performed) was significantly longer in the SEQ compared with the CON group (79.9 vs. 43.4 days, $\mathrm{p}=0.00)$.

\section{Toxicity (table 2)}

Three treatment-related deaths occurred in the SEQ-group (due to hematotoxicity), whereas no such serious complication was found in patients assigned to the CON group. Acute local toxicity was generally moderate in both groups, except for severe mucositis, which affected $54 \%$ of patients receiving hyperfractionated radiotherapy in the CON-group compared with $44 \%$ in SEQ group (n.s.). Regarding late toxicity, lymph edema, mouth dryness and dysphagia were the most frequently observed grade III and IV side effects in both groups, as summarized in table 2 . The frequency of grade I or II acute toxicity was higher,

Table 3: Comparison of responses in dependency on the schedule

\begin{tabular}{|c|c|c|c|c|c|c|c|c|}
\hline & \multicolumn{4}{|l|}{ SEQ $(n=70)$} & \multicolumn{4}{|c|}{$\operatorname{CON}(n=52)$} \\
\hline & \multicolumn{2}{|c|}{$\begin{array}{l}\text { After } 2 \text { cycles } \\
\text { chemotherapy }\end{array}$} & \multicolumn{2}{|c|}{$\begin{array}{l}\text { After chemotherapy and } \\
\text { radiotherapy }\end{array}$} & \multicolumn{4}{|c|}{ After chemotherapy and radiotherapy } \\
\hline & Primary (\%) & Nodes (\%) & Primary (\%) & Nodes (\%) & Primary (\%) & $\mathrm{P}^{2)}$ & Nodes (\%) & $\mathrm{P}^{2)}$ \\
\hline Evaluable & $93(65 / 70)$ & $93(65 / 70)$ & $89(62 / 70)$ & $86(60 / 70)$ & $98(5 \mathrm{I} / 52)$ & 0.02 & $98(5 \mathrm{I} / 52)$ & 0.00 \\
\hline Complete response I) (CR) & 11 & 7 & 41 & 33 & 46 & n.s. & 58 & n.s. \\
\hline Partial remission I) (PR) & 51 & 51 & 26 & 33 & 44 & n.s. & 37 & n.s. \\
\hline $\mathrm{CR}$ and $\mathrm{PR}$ & 63 & 59 & 67 & 66 & 90 & n.s. & 94 & n.s. \\
\hline NC, Progressive disease & 30 & 33 & 21 & 20 & 8 & n.s. & 4 & n.s. \\
\hline
\end{tabular}

1) $\mathrm{WHO}$

2) compared with response in the SEQ-group after chemotherapy and radiotherapy 
Table 4: Comparison of treatment results between the two groups

\begin{tabular}{lccc}
\hline & SEQ & CON & P \\
\hline Overall survival (5 ys.) & $14.7 \%$ & $23.7 \%$ & 0.11 \\
Median overall survival & 14.6 months & 20.1 months & 0.08 \\
Disease-free survival $(5$ ys.) & $19.8 \%$ & $31.4 \%$ & $41.0 \%$ \\
Progression free survival $(5$ ys.) years) & $17.6 \%$ & 15.0 months & 0.03 \\
Median time to (local) progression & 10.7 months & \\
\hline
\end{tabular}

1) WHO 2) compared with response in the SEQ-group after chemotherapy and radiotherapy

as expected, in the CON group. Occurrences of grade I or II late toxicity proved to be equally distributed.

\section{Response}

The complete primary tumor remission (CR) rates were $41 \%$ in the SEQ vs. $46 \%$ in the CON group, and the partial remission (PR) rates were $26 \%$ vs. $44 \%$, respectively. Response in the lymph nodes was also less frequent in the SEQ than the CON group ( $86 \%$ vs. $98 \%$ ). A survey of the response rates in primary tumors and involved lymph nodes is given in table 3 . The overall differences (CR + PR $+\mathrm{PD} / \mathrm{NC}$ ) in response (CON vs. SEQ) were significant for both the primary tumors $(\mathrm{p}=0.02)$ and the lymph nodes $(\mathrm{p}=0.00)$, but the single endpoints (CR or PR or PD/NC) did not show significant between-group differences. Calculations were performed on the total number of eligible patients, but it is notable that the proportion of non-evaluable patients was considerably higher in the SEQ group (8/70 versus $1 / 52)$ and included four patients receiving no radiotherapy at all (three were treatment-related deaths). This difference in treatment failure and disruption is a further criterion in favour of the CON group, but it is not encompassed by the response analysis.

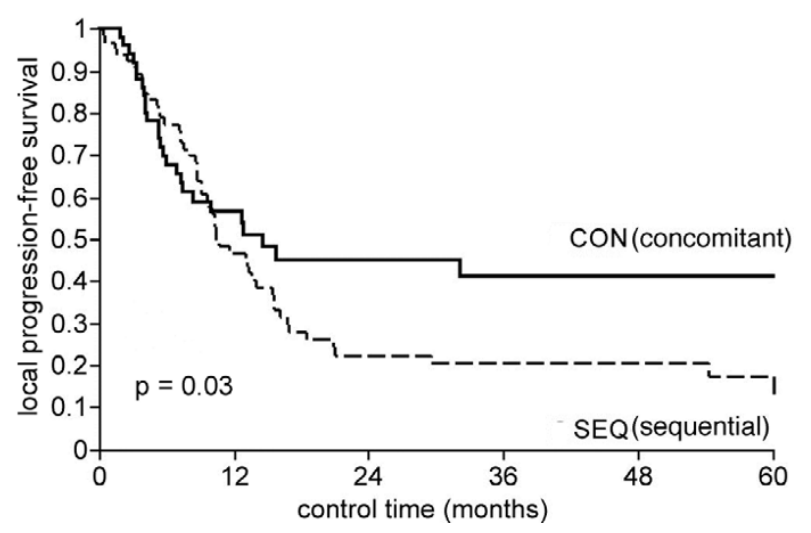

Figure 2

Kaplan-Meier survival curves with locoregional control, i.e. detecting time-to-local progression, in relation to the type of protocol.

\section{Local control and survival}

Calculations were performed on the eligible patients and the results are summarised in table 4 . The 5-year local control rate (local-progression-free survival) was significantly lower in the SEQ-group than in the CON-group according to fig. 2 ( $17.6 \%$ vs $41 \%, \mathrm{p}=0.03$ with 51 patients vs. 26 patients relapsing locally). Among the patients achieving complete remission, $29 \%$ had recurrences of the primary and $24 \%$ in the lymph nodes in the CON group after 5 years, compared with $55 \%$ and $48 \%$ in the SEQ group. The most common site of recurrence in the SEQ-group was locoregional (90\% of progressive patients), whereas distant metastases (DM) alone or combined with local failure (LF) occurred very rarely $(4 \%$ or $6 \%, \mathrm{DM} \pm \mathrm{LF})$. In contrast, only $73 \%$ had LF alone in the CON-group, whereas $17 \%$ of the patients suffered from initial DM $( \pm$ $\mathrm{LF}$ ). Overall, fewer patients in the SEQ-group than in the CON-group presented with DM ( \pm LF) (actuarially $18 \%$ or $26 \%$ after 5 years, 6 patients vs. 10 patients). Secondary cancers occurred in both groups with similar frequencies (7\% and $8 \%)$.

The actuarial disease-specific survival curves (fig. 3, table 4) were not statistically different (5 years: $19.8 \%$ and $31.4 \%$, with tumor-related deaths of 52 patients and 30 patients), but showed a trend in favour of the CON-group $(\mathrm{p}=0.08)$. The overall survival curves showed the same tendency, with 3 -year survival rates of $30 \%$ vs. $21 \%$ and 5 year survival rates of $24 \%$ vs. $15 \%$ in favour of the CONgroup ( $\mathrm{p}=0.11,56$ vs. 36 patients dying from all causes) (fig. 4).

\section{Predictors of outcome}

The prognostic factors that had proved significant in univariate analysis were subjected to multivariate analysis, which showed that T-categories ( $\leq \mathrm{T} 3$ vs. T4, p $<0.001)$ and $\mathrm{N}$-categories ( $\leq \mathrm{N} 2 \mathrm{a}$ vs. N2b, N3, p $<0.01$ ) were significantly correlated with locoregional control. The type of treatment protocol had a borderline significant relationship ( $\mathrm{p}=0.06$ ) with local treatment outcome. Performance status, RTOG stage and radiation dose proved not to be significantly correlated in the multivariate analysis. 


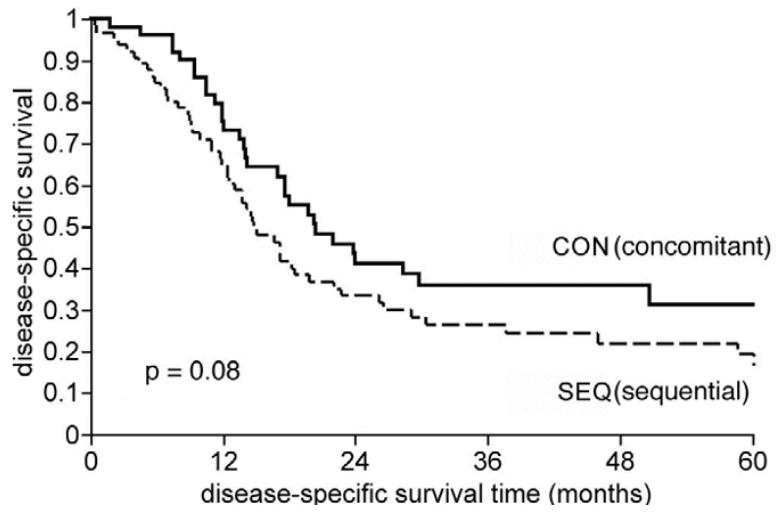

Figure 3

Kaplan-Meier disease-specific survival curves, i.e. considering tumor-related deaths, in relation to the type of protocol.

With regard to disease-specific survival, a multivariate analysis of the significant prognostic factors from the univariate analysis revealed T-stage ( $\leq \mathrm{T} 3$ vs. T4, $\mathrm{p}<0.001$ ), $\mathrm{N}$-stage ( $\leq \mathrm{N} 2 \mathrm{a}$ vs. N2b, N3, $<<0.001)$, performance status ( $\mathrm{p}=0.04)$ and treatment protocol (SEQ vs. CON, $\mathrm{p}<$ 0.01 ) as significant predictors. The total dose showed borderline significant correlation ( $\mathrm{p}=0.06)$ with disease-specific survival, whereas there was no significant correlation for the RTOG stage. $\mathrm{N}$-stage ( $\mathrm{p}=0.00)$, treatment protocol $(\mathrm{p}=0.03)$ and radiation dose $(\mathrm{p}=0.05)$ proved significant for overall survival.

\section{Discussion}

Radiochemotherapy is becoming increasingly established as a standard method for treating inoperable head and neck carcinomas, but the optimal scheme is still under discussion. One reason is the large variation in risk factors among patient groups.

It has been found that the percentage of local control after 5 years following a treatment regimen consisting solely of radiotherapy is improved by adding chemotherapy (5-FU, cisplatin etc.), which has conferred survival benefit in a number of trials. Several studies have failed to demonstrate any influence on the frequency of systemic dissemination.

We evaluated two different radiochemotherapeutic approaches to patients with locally advanced head and neck cancer. Prognostic factors were more or less equally distributed in the two groups. Clearly, the groups differed in treatment parameters in concerning radiation dose, cytotoxic drugs, treatment time, etc. Therefore, it might be difficult to estimate the influence of a single parameter on treatment outcome. In our study, improvements in dis- ease staging and support measures could not have been major contributors. Treatment technique, treatment planning and radiation therapy technology were not changed significantly in our institution during the time encompassing the studies on the two groups.

In general, our results suggest that concomitant radiochemotherapy is superior with respect to local control, but not unequivocally with respect to distant metastases or overall survival. The regenerative response of clonogens may weaken the contribution of induction chemotherapy to locoregional control. In addition, the toxicity of the applied dose-intense chemotherapy often impaired the regular administration of radiotherapy within the SEQgroup (an attribute of the sequential scheme), resulting in treatment interruptions that led to a mean treatment time twice as long as in the CON-group. This shows that the impairment in quality of life following sequential treatment - in addition to worse results - may be greater than the adverse effects of concomitant radiochemotherapy.

The 5-year overall survival rates (15\% vs. $24 \%$ ) and 5 -year disease-free survival rates ( $20 \%$ vs. $31 \%$ ) are relatively low in both groups, obviously because all except one patient suffered from stage IV disease, and a high proportion of patients in both groups had unfavourable prognostic indicators (see table 1). Other investigators report similarly low overall survival rates for patients with comparably unfavourable prognostic factors $[4,12,13]$.

Better treatment results are achieved with selected collectives. Regarding recent prospective trials, 3-year local control rates of $50-70 \%$ are not uncommon in patients treated with irradiation and concurrent chemotherapy (with conventional or altered fractionation) [14]. For example, Dinges et al. [10] reported a high disease-free survival rate of $59 \%$ and a local control rate of $72 \%$ after 4 years using the treatment schedule applied in the CONgroup of our analysis. Overall, most of the published studies [14] report 2-3 year overall survival rates of around $20-30 \%$ following radiotherapy and $40-50 \%$ following concomitant radiochemotherapy [3,14-16], comparable to our $30-40 \%$ 2-3-year survival rate after concomitant RCT (fig. 5). The difference of about $10 \%$ can easily be explained by the poor prognostic indicators of our (rather unselected) patient groups. Note that in a large meta-analysis with more than 10,000 patients, the survival rates for any kind of locoregional treatment plus chemotherapy (including radical surgery with adjuvant or neoadjuvant chemotherapy) were not higher than $36 \%$ after 5 years [5], compared to $24 \%$ in our CON group.

Patients with unresectable head and neck tumors have a limited risk of death from distant metastases, at least in comparison to patients suffering from gastrointestinal 


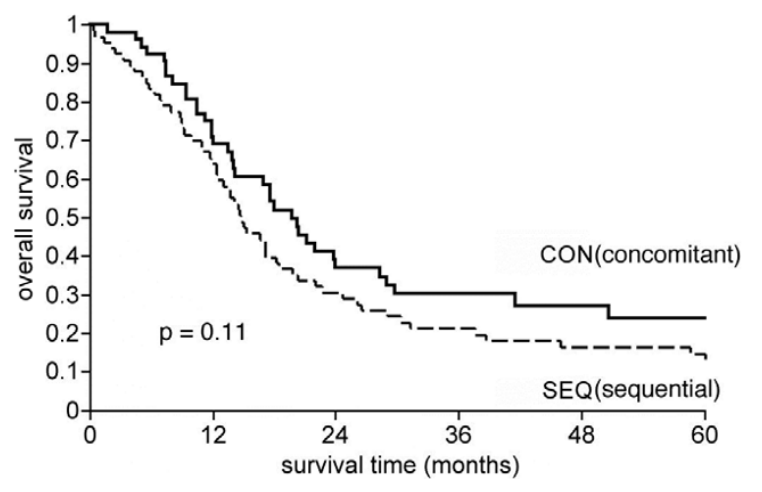

Figure 4

Kaplan-Meier overall survival curves, i.e. considering all causes of death, in relation to the type of protocol.

malignancies. Studies based on autopsies have verified that $40 \%$ of patients with head and neck cancers died from local relapses without evidence of distant metastases or secondary primary tumors (32\%), whereas only $5 \%$ of patients died from distant metastases alone [17].

Theoretically, neoadjuvant chemotherapy can benefit a few patients directly (by tumor regression in responders), but may also reduce the incidence of distant metastases, especially in collectives with known risk factors. In a number of clinical studies of induction chemotherapy $[18]$, neither significant downstaging nor a decreased incidence of distant metastases was observed. In a meta-analysis, Pignon found no significant benefit from induction chemotherapy as opposed to concomitant chemotherapy in head and neck cancer [5]. In our study, local failures were the most frequent causes of death, and so far as local control is concerned we found a markedly better outcome in the CON-group. On the other hand, patients with metachronous distant metastases were less frequent in the SEQ-group.

Randomised studies comparing induction chemotherapy with subsequent radiotherapy against radiotherapy alone $[2,15]$ have failed to showany significant advantage in terms of overall survival and disease-free survival. On the other hand, concomitant application of cytostatic drugs has resulted in significant improvement, as shown in several randomised trials, independently of fractionation $[2,3,19]$. Radiochemotherapy can even improve on radiotherapy alone with dose escalation [4]. In other studies, a randomised comparison between hyperfractionated doseescalated radiotherapy and hyperfractionated radiotherapy with concomitant chemotherapy showed only slight advantages for the combined arm [20]. Only a few studies have been conducted on randomised comparisons between concomitant radiochemotherapy (with moderate chemotherapy dosages) and sequential radiochemotherapy (with systemically effective chemotherapy dosages). In this regard, Adelstein [2] reported a significant increase in the local control rate using concomitant RCT. Taylor [21] achieved significantly better disease-free survival, although overall survival was not significantly increased.

\section{Conclusion}

In comparison with concomitant radiochemotherapy, induction chemotherapy followed by radiotherapy has lost popularity. This was confirmed by our retrospective analysis, i.e. treatment with induction chemotherapy proved inferior with regard to applicability and efficacy and resulted in unsatisfactory survival rates and low patient compliance. Although induction chemotherapy might result in higher systemic effectiveness, inferior locoregional control dominates the clinical outcome. Schemes using simultaneous chemotherapy and hyperfractionated radiotherapy generally show greater acute toxicity and better overall survival, tumour specific survival, disease-free survival and local control if patient selection is appropriate. To optimise the combination of both modalities (radiotherapy and chemotherapy) the aim must be to achieve higher local effectiveness together with tolerable acute and late toxicities.

\section{Abbreviations}

Head-and-neck carcinoma (H\&N); sequential protocol (SEQ); cisplatin (CDDP); folinic acid (FA); 5-fluorouracil (5-FU); concomitant protocol (CON); mitomycin (MMC); local control (LC); disease-specific survival (DSS); MTX (methotrexate); time-to-progression (TTP); complete remission (CR); partial remission (PR); distant metastases (DM); local failure (LF).

\section{Competing interests}

The author(s) declare that they have no competing interests.

\section{Authors' contributions}

R.G. performed the radiotherapy, analysed and interpreted the data, and drafted the tables, figures and manuscript. B. H. performed the chemotherapy and critically revised the manuscript. W. T. performed parts of the statistical analysis and contributed to the figures. H. R. was the consultant for chemotherapy and established induction chemotherapy plus RCT. R. F. performed the followup and was the consultant for diagnostics. V. B. established the concomitant RCT and was the consultant for radiotherapy. P. W. supervised the acquisition, analysis and interpretation of data, and conceived and revised the manuscript. 


\section{References}

I. Stuschke M, Thames $\mathrm{H}$ : Hyperfractionation: where do we stand? Radiother Oncol 1998, 46:131-33.

2. Adelstein DJ, Sharan VM, Earle AS, Shah AC, Vlastou C, Haria CD, Damm C, Carter SG, Hines JD: Concomitant versus sequential combined technique therapy for squamous cell head and neck cancer. Cancer 1990, 65:1685-9I.

3. Wendt TG, Grabenbauer GG, Rodel CM, Thiel HJ, Aydin H, Rohloff $\mathrm{R}$, Wustrow TP, Iro H, Popella C, Schalhorn A: Concomitant radiochemotherapy versus radiotherapy alone in advanced head and neck cancer: a randomized multicenter study. I Clin Oncol 1998, 16: | 3 18-24.

4. Budach V, Stuschke M, Budach W, Baumann M, Geismar D, Grabenbauer G, Lammert I, Jahnke K, Stueben G, Herrmann T, Bamberg M, Wust P, Hinkelbein W, Wernecke KD: Hyperfractionated accelerated chemoradiation with concurrent fluorouracil-mitomycin is more effective than dose-escalated hyperfractionated accelerated radiation therapy alone in locally advanced head and neck cancer: final results of the Radiotherapy Cooperative Clinical Trials Group of the German Cancer Society 95-06 Prospective Randomized Trial. Clin Oncol 2005, 23: I I25-II35.

5. Pignon JP, Bourhis J, Domenge C, Designe L: Chemotherapy added to locoregional treatment for head and neck squamous-cell carcinoma: three meta-analyses of updated individual data. MACH-NC Collaborative Group. Meta-Analysis of Chemotherapy on Head and Neck Cancer. Lancet 2000, 355:949-55

6. Spiessl B, Beahrs OH, Hermanek P, Hutter RV, Scheibe O, Sobin LH, Wagner G: TNM atlas. In Illustrated guide to the TNM/pTNM classification of malignant tumors (ed 3, 2nd rev) Berlin, Germany, SpringerVerlag; 1992.

7. Cox JD, Stetz J, Pajak TF: Toxicity criteria of the Radiation Therapy Oncology Group (RTOG) and the European Organization for Research and Treatment of Cancer (EORTC). Int J Radiat Oncol Biol Phys 1995, 3 I: I 34 I-46.

8. Rubin P, Constine LS, Fajardo LF, Phillips TL, Wasserman TH: Late Effects Working Group. Overview. Late Effects of Normal Tissues (LENT) scoring system. Int J Radiat Oncol Biol Phys 1995, 30: $1041-42$

9. Miller $A B$, Hoogstraten $B$, Staquet $M$, Winkler $A$ : Reporting results of cancer treatment. Cancer I98I, 47:207-I4.

10. Dinges S, Budach V, Stuschke M, Budach W, Boehmer D, Schrader M, Jahnke K, Sack H: Chemo-radiotherapy for locally advanced head and neck cancer - long-term results of a phase II trial. Eur J Cancer 1997, 33: I I52-55.

II. Kaplan EL, Meier P: Nonparametric estimation from incomplete observations. J Am Stat Assoc 1958, 53:457-8I.

12. Wibault $P$, Bensmaine MA, de Forni M, Armand JP, Tellez Bernal E, Guillot T, Recondo G, Domenge C, Janot F, Borel C, Luboinski B, Eschwege F, Cvitkovic E: Intensive concomitant chemoradiotherapy in locally advanced unresectable squamous cell carcinoma of the head and neck: a phase II study of radiotherapy with cisplatin and 7 -week continuous infusional fluorouracil. J Clin Oncol 1996, | 4: | |92-200.

13. Paccagnella A, Orlando A, Marchiori C, Zorat PL, Cavaniglia G, Sileni VC, Jirillo A, Tomio L, Fila G, Fede A, et al.: Phase III trial of initial chemotherapy in stage III or IV head and neck cancers: a study by the Gruppo di Studio sui Tumori della Testa e del Collo. J Natl Cancer Inst 1994, 86:265-72.

14. Brizel DM: Radiotherapy and concurrent chemotherapy for the treatment of locally advanced head and neck squamous cell carcinoma. Sem Radiat Oncol 1998, 8:237-46.

15. Merlano M, Benasso M, Corvo R, Rosso R, Vitale V, Blengio F, Numico G, Margarino G, Bonelli L, Santi L: Five-year update of a randomized trial of alternating radiotherapy and chemotherapy compared with radiotherapy alone in treatment of unresectable squamous cell carcinoma of the head and neck. I Natl Cancer Inst 1996, 88:583-89.

16. Fu KK, Phillips TL, Silverberg IJ, Jacobs C, Goffinet DR, Chun C, Friedman MA, Kohler M, McWhirter K, Carter SK: Combined radiotherapy and chemotherapy with bleomycin and methotrexate for advanced inoperable head and neck cancer: Update of a Northern California Oncology Group randomized trial. J Clin Oncol I987, 5:|410-18.
17. Slootweg PJ, Hordijk G], Koole R: Autopsy findings in patients with head and neck squamous cell cancer and their therapeutic relevance. Eur J Cancer B Oral Oncol 1996, 32B:4I3-I5.

18. Adelstein DJ, Tan EH, Lavertu P: Treatment of head and neck cancer: the role of chemotherapy. Crit Rev Oncol Hematol 1996, 24:97-II6.

19. Brizel DM, Albers ME, Fisher SR, Scher RL, Richtsmeier WJ, Hars V, George SL, Huang AT, Prosnitz LR: Hyperfractionated irradiation with or without concurrent chemotherapy for locally advanced head and neck cancer. $N$ Engl J Med 1998 338: 1798-804.

20. Staar S, Rudat V, Stuetzer H, Dietz A, Volling P, Schroeder M, Flentje $M$, Eckel HE, Mueller RP: Intensified hyperfractionated accelerated radiotherapy limits the additional benefit of simulaneous chemotherapy - results of a multicentric randomized German trial in advanced head-and-neck cancer. Int J Radiat Oncol Biol Phys 200I, 50(5): I I6I-7I.

2I. Taylor SG 4th, Murthy AK, Vannetzel JM, Colin P, Dray M, Caldarelli DD, Shott S, Vokes E, Showel JL, Hutchinson JC: Randomized comparison of neoadjuvant cisplatin and fluorouracil infusion followed by radiation versus concomitant treatment in advanced head and neck cancer. J Clin Oncol 1994, I 2:385-95.

\section{Pre-publication history}

The pre-publication history for this paper can be accessed here:

http://www.biomedcentral.com/1471-2407/6/30/prepub
Publish with Biomed Central and every scientist can read your work free of charge

"BioMed Central will be the most significant development for disseminating the results of biomedical research in our lifetime. "

Sir Paul Nurse, Cancer Research UK

Your research papers will be:

- available free of charge to the entire biomedical community

- peer reviewed and published immediately upon acceptance

- cited in PubMed and archived on PubMed Central

- yours - you keep the copyright
BioMedcentral 